\title{
4D Analytical STEM with the pnCCD
}

M. Huth ${ }^{1}$, M. Simson ${ }^{1}$, R. Ritz ${ }^{1}$, H. Ryll ${ }^{2}$, G.T. Martinez ${ }^{3}$, J. Lozano ${ }^{3}$, P.G. Bruce ${ }^{3}$, P.D. Nellist ${ }^{3}$, R. Sagawa $^{4}$, Y. Kondo ${ }^{4}$ and H. Soltau ${ }^{1}$

1. PNDetector GmbH, Otto-Hahn-Ring 6, München, Germany.

2. PNSensor GmbH, Otto-Hahn-Ring 6, München, Germany.

3. Department of Materials, University of Oxford, Oxford, UK.

4. JEOL Ltd., 3-1-2 Musashino Akishima Tokyo, Japan.

The availability of the pnCCD (S)TEM camera, combining ultra-fast direct detection at single electron sensitivity with a radiation hard diode-like technology, inspired the development of various four dimensional STEM imaging techniques (4D STEM). Here, a focused beam of electrons is applied to a sample in a two dimensional (2D) raster pattern. At each point, a 2D image is captured which intrinsically contains bright field (BF), dark field (DF), and high angle annular dark field (HAADF) signals. In total, a $4 \mathrm{D}$ dataset is recorded allowing a comprehensive analysis and enabling a number of techniques such as strain analysis, magnetic domain mapping, scanning electron diffraction, and electron ptychography. The key parameters of the pnCCD (S)TEM camera, which are particularly beneficial for 4D STEM techniques are: (a) Fast acquisition of data to minimize effects of beam and sample drift, and beam induced damage to the sample; (b) a large number of detector pixels to precisely discriminate between diffraction spots or to determine the position of a bright field disk with high accuracy; and (c) a sufficiently radiation hard detector compatible with high electron rates as used in scanning electron diffraction experiments. Instead of counting, the unique diode technology with a fully depleted sensor enables the analytical measurement of all incident electrons.

The camera is a complete system including a data acquisition computer and software allowing a versatile live visualization and full raw data access. With a pixel resolution of $264 \times 264$, the pnCCD (S)TEM camera provides ultra-fast acquisition of 2D camera images [1]. Routinely, the readout speed is 1000 frames per second (fps) in full frame mode and can be further increased up to 8000 fps by binning and windowing. The camera head is available as fixed bottom mount or retractable design and can be operated with electron energies ranging from $20 \mathrm{keV}$ to $300 \mathrm{keV}$. By operating the camera in one of three predefined operation modes [2], optimum results are guaranteed for a wide range of experimental conditions. The acquired information rich 4D datasets can be analyzed in a number of ways, such as virtual diffraction images, which can be calculated and the phase information can be extracted.

The outstanding capabilities of the pnCCD (S)TEM camera were demonstrated in numerous experiments at various TEMs. As first example, a monolayer of graphene was imaged on a JEOL-ARM 200F operated at $80 \mathrm{kV}$ and a probe current of $\sim 4.7 \mathrm{pA}$ and an electron dose of $3.7 \cdot 10^{4} \mathrm{e}^{-} / \AA$ on the sample, using $2 \mathrm{x}$ binning at $2000 \mathrm{fps}$ readout speed. Simultaneously, a conventional ADF image was recorded for comparison. As shown in Figure 1, the contrast can be drastically improved by calculating the phase contrast image from the 4D data set. The second example shows a battery material; a charged $\mathrm{Li}_{1.2} \mathrm{Mn}_{0.6} \mathrm{Ni}_{0.2} \mathrm{O}_{2}$ particle, a Li-rich layered oxide cathode, which has improved capacity and avoids expensive Co, compared to current cathode materials. Both, their advantages and the processes that affect their utilization are inherently local. Measurements of charged samples require special care as phase transformations may be induced by the electron beam. With the pnCCD (S)TEM camera it is possible to obtain high contrast images at a very low dose using sub pA beam currents, enabling imaging of low $\mathrm{Z}$ 
materials in a low dose setting. Figure 2 shows an example of a Li-rich layered oxide cathode, where the phase contrast method visualizes the atomic arrangement.

\section{References:}

[1] Ryll, H. et al, Journal of Instrumentation 11 (2016).

[2] Schmidt J. et al, Journal of Instrumentation 11 (2016).

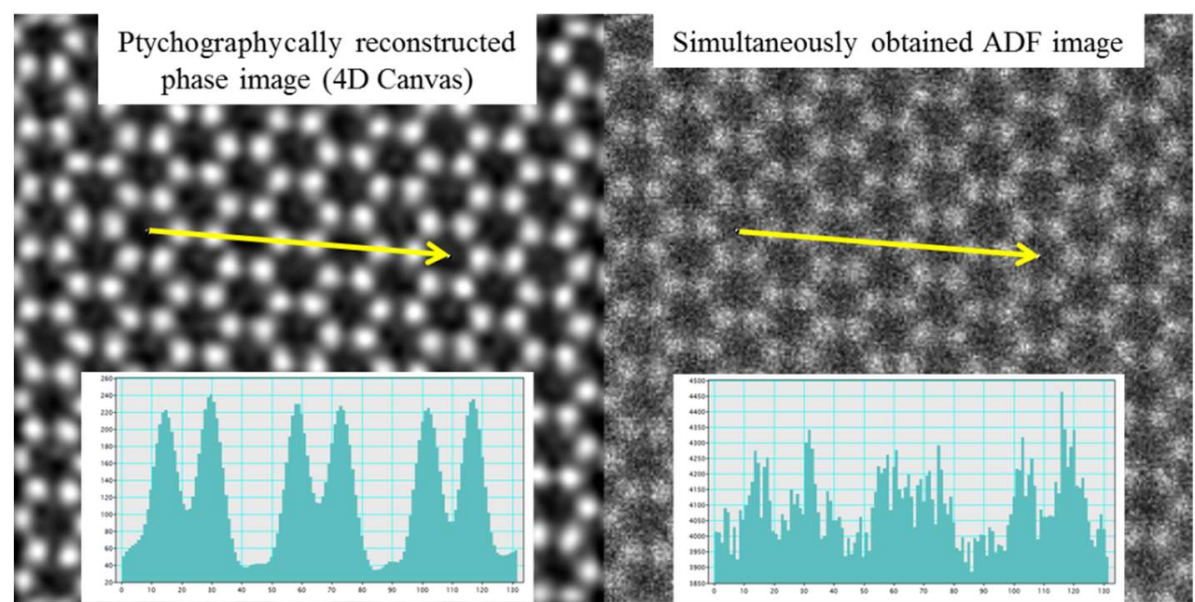

Figure 1. Monolayer graphene recorded with $2000 \mathrm{fps}$ on a JEOL ARM200F at $80 \mathrm{kV}$. Probe current was $4.7 \mathrm{pA}$ with an electron dose of $3.7 \cdot 10^{4} \mathrm{e}^{-/} \AA$. The comparison of the phase image (left) and the simultaneous acquired ADF image illustrates the enhanced SNR and contrast of the 4D STEM technique. Data recorded and analyzed by JEOL Japan.

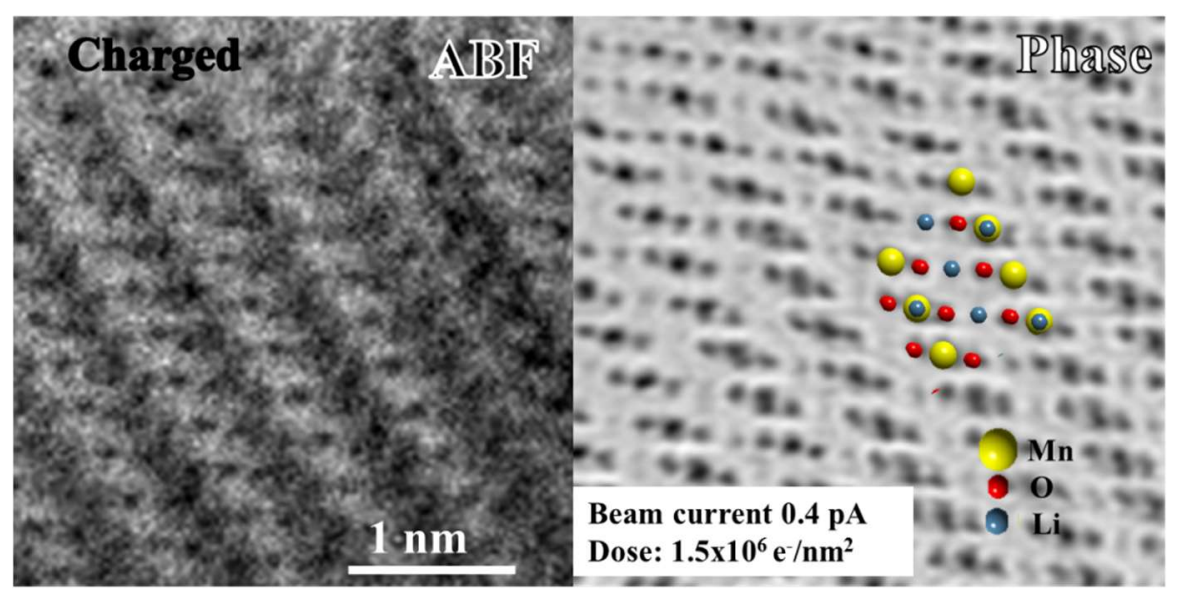

Figure 2. Sample of charged $\mathrm{Li}_{1.2} \mathrm{Mn}_{0.8} \mathrm{Ni}_{0.2} \mathrm{O}_{2}$ recorded with 4000 fps on a JEOL ARM200F at $200 \mathrm{kV}$. While barely visible in the $\mathrm{ABF}$ image, the phase image reveals the contrast due to the transition metals, oxygen and also in the alkali layer. The schematic overlay shows the structure and elemental arrangement. Data recorded and analyzed by University of Oxford, Department of Materials. 